\title{
Social Percolation and the Influence of Mass Media
}

Ana Proykova ${ }^{1}$ and Dietrich Stauffer

Institute for Theoretical Physics, Cologne University, D-50923 Köln, Euroland

${ }^{1}$ Department of Atomic Physics, University of Sofia, Sofia-1126, Bulgaria

Abstract: Mass media shift the percolative phase transition observed in the marketing model of Solomon and Weisbuch.

Keywords: percolation, external field, customers, cinema

"Social percolation" [1] was invented to describe how a new product can penetrate a consumer market by word-of-mouth. Imagine a new movie comes out, and person $i$ goes to the cinema if and only if (s)he hears from neighbours that the movie quality $q$ is above the own quality expectations $p_{i}$. Initially, $q=1 / 2$, and the $p_{i}$ are distributed randomly between 0 and 1 . If a movie is a success, the next movie has its quality $q$ lowered by 0.001 ; if the movie is a flop, the next movie will be better by 0.001 . In this way, $q$ moves towards the percolation threshold $p_{c}(=0.593$ on the square lattice): self-organized criticality [2].

It has been suggested [3] to include the effect of mass media through advertizing, in addition to the traditional word-of-mouth propaganda from the neighbours $\mathbb{1 1}$, 2, 田. In percolation theory such a role is played by the ghost field $h$ which couples each site to a ghost site with probability 1 $\exp (-h)$, just as telephone links via sattelite may keep together a widely scattered family. This ghost field $h$ plays the role of a magnetic field in Ising models and rounds the phase transition at the critical point. However, since social percolation is a dynamic process, the analogy of the present simulations with magnetic or ghost fields is not complete.

We thus assume that with a certain probability $h$ every site $i$ initially is informed about the quality $q$ of a new movie; the informed sites with own expectation $p_{i}$ below $q+0.001$ visit the movie. Then they tell their neighbours, the normal social percolation process starts, the mass media no longer play any role. (The number 0.001 is added to $q$ to account for slightly different convincing powers of movie revies compared with neighbour opinions.)

When is a movie called a success, causing $q$ to decrease for the next film? In traditional social percolation without mass media, the criterion was the 


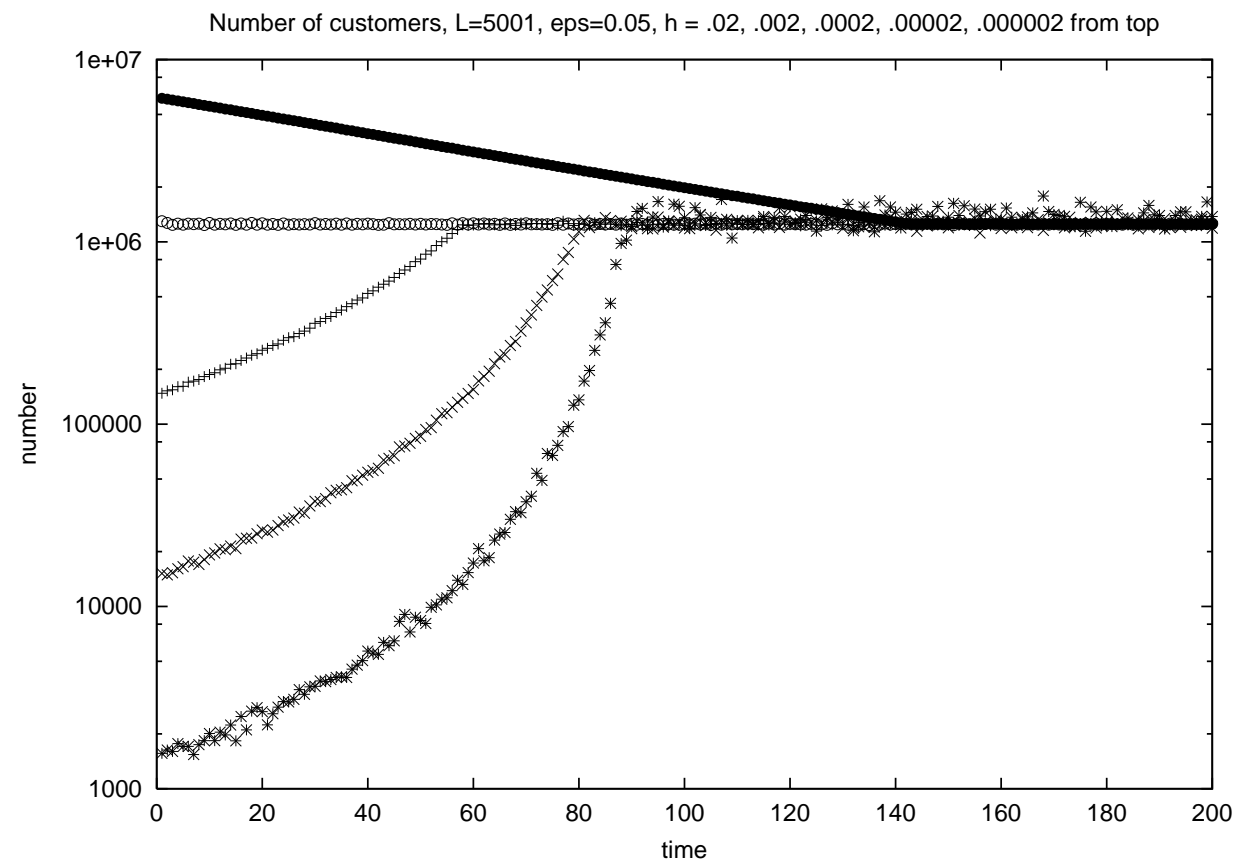

Figure 1: Variation with time of the number of customers for various $h=$ 0.000002 to 0.02 at fixed $\epsilon=0.05$, again from 20 lattices $5001 \times 5001$.

existence of a cluster of movie goer extending from one side of the lattice (Hollywood) to the other (New York). With a ghost field one always has an infinite cluster, just as with a magnetic field one gets a finite magnetization. In our case, through mass media one single site on the border opposite to the initially informed border of the lattice can visit the movie. Thus a percolating cluster no longer means success. Instead, we define a movie to be a success if at least a fraction $\epsilon$ of all people go to this movie; typically, $\epsilon$ is five percent. We no longer have to initialize one lattice border as being informed; those sites which are convinced by the mass media (typically $h=0.0002$ ) are the starting sites of the word-of-mouth propaganda.

We start with one movie having quality $q$; depending on whether or not the threshold fraction $\epsilon$ of movie visits is reached, the quality is changed to 0.499 or 0.501 . Then this movie is put onto the market, with a new initial distribution of people convinced my mass media. This process is repeated again and again for 200 movies, and during this iteration the resulting quality 


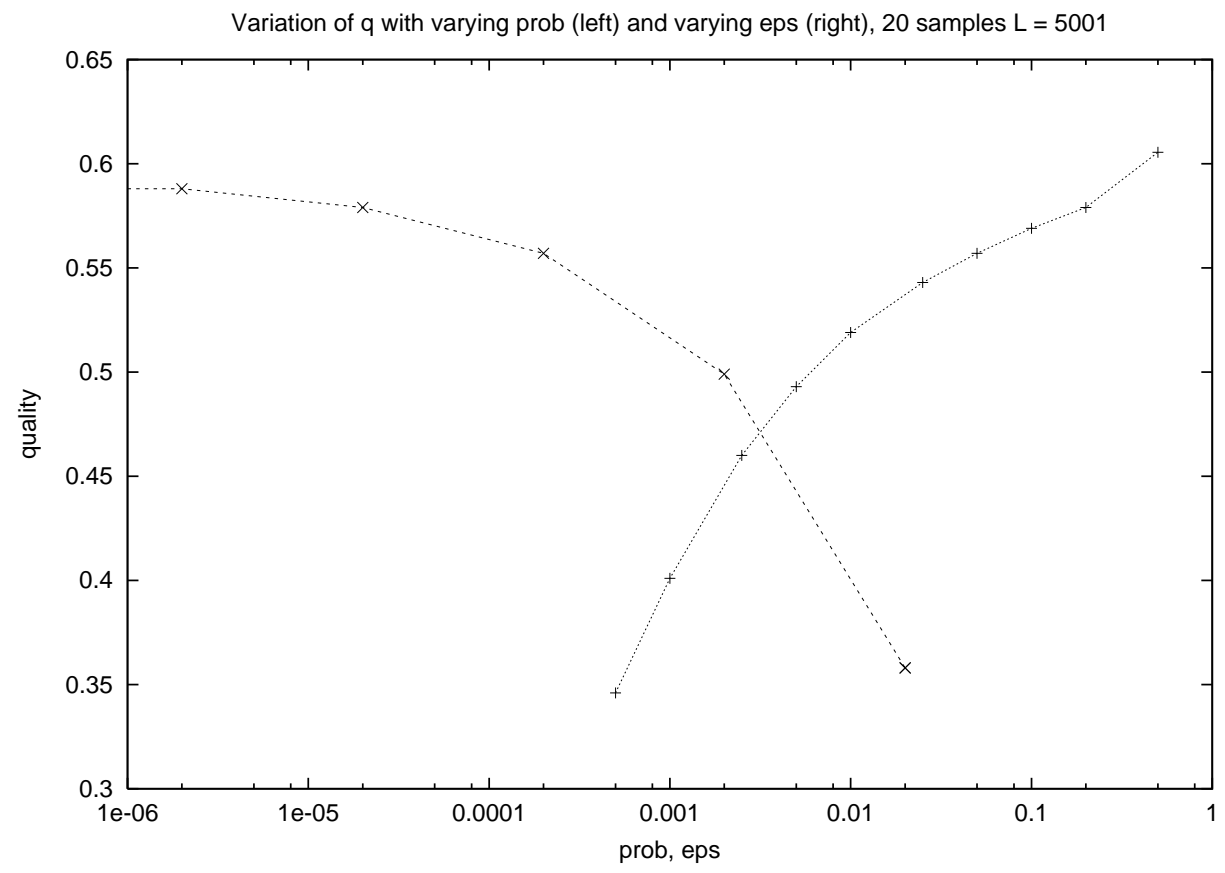

Figure 2: Variation with $h$ for fixed $\epsilon=0.05$ (left curve) and variation with $\epsilon$ for fixed $h=0.0002$ of the final quality $q_{f}$, averaged over 20 square lattices $5001 \times 5001$.

$q$ first changes linearly in time until it reaches its stationary value $q_{f}$ and fluctuates about this value. To avoid the problems discussed by Huang [5], we do not change the $p_{i}$ during the simulation of one movie; each new movie gets a new distribution of $p_{i}$.

Fig. 1 shows how the number of customers in a market of 25 million varies as a function of time (= number of iterations = number of new movies introduced to the market). The quality $q$ as a function of time starts from zero and moves linearly to its stationary value about which it fluctuates thereafter. Fig. 2 shows this final quality $q$ as a function of $h$ and $\epsilon$. Markets above a million show no significant size effects, Fig.3. In the stationary state, the distribution of the numbers of customers shows no clear distinction between hits and flops, in agreement with reality [6].

We also coupled the advertising success $h$ with the quality expectations by assuming a probability $\left(1+p_{i}\right) h$ or $\left(2-p_{i}\right) h$ to believe initially the mass 


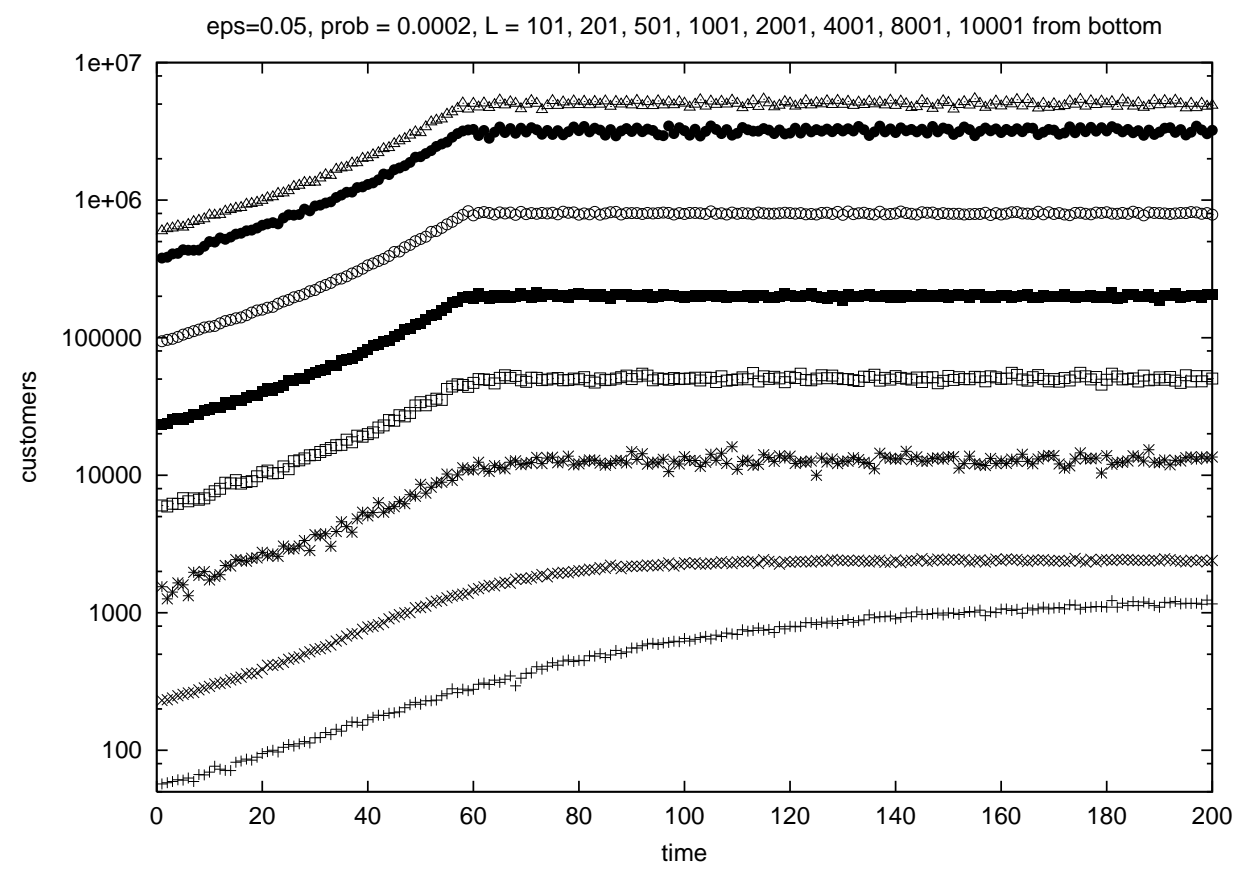

Figure 3: Search for size effects for $101 \times 101$ up to $10001 \times 10001$.

media, instead of just $h$. However the only role of this coupling, Fig.4, is the increase of the initial population of buyers (movie watchers) and the decrease of the time, necessary to convince reasonable amount of people to go. Most probably this is good for the trading (movie-making) company.

The plot of $L=2001$ with various $\epsilon$ (not shown) shows the same pattern already observed in Fig. 1 for the decoupled case $(L=5001)$. Again the role of coupling the advertisment to the internal expectations is the increase of the initial number of customers.

In summary we see that a moderate amount of advertising decreases the time after which the number of customers and the quality of the product reaches the stationary state (provided the cost of advertising is smaller than the resulting profit).

This work was supported by the Sofia-Cologne university partnership. 


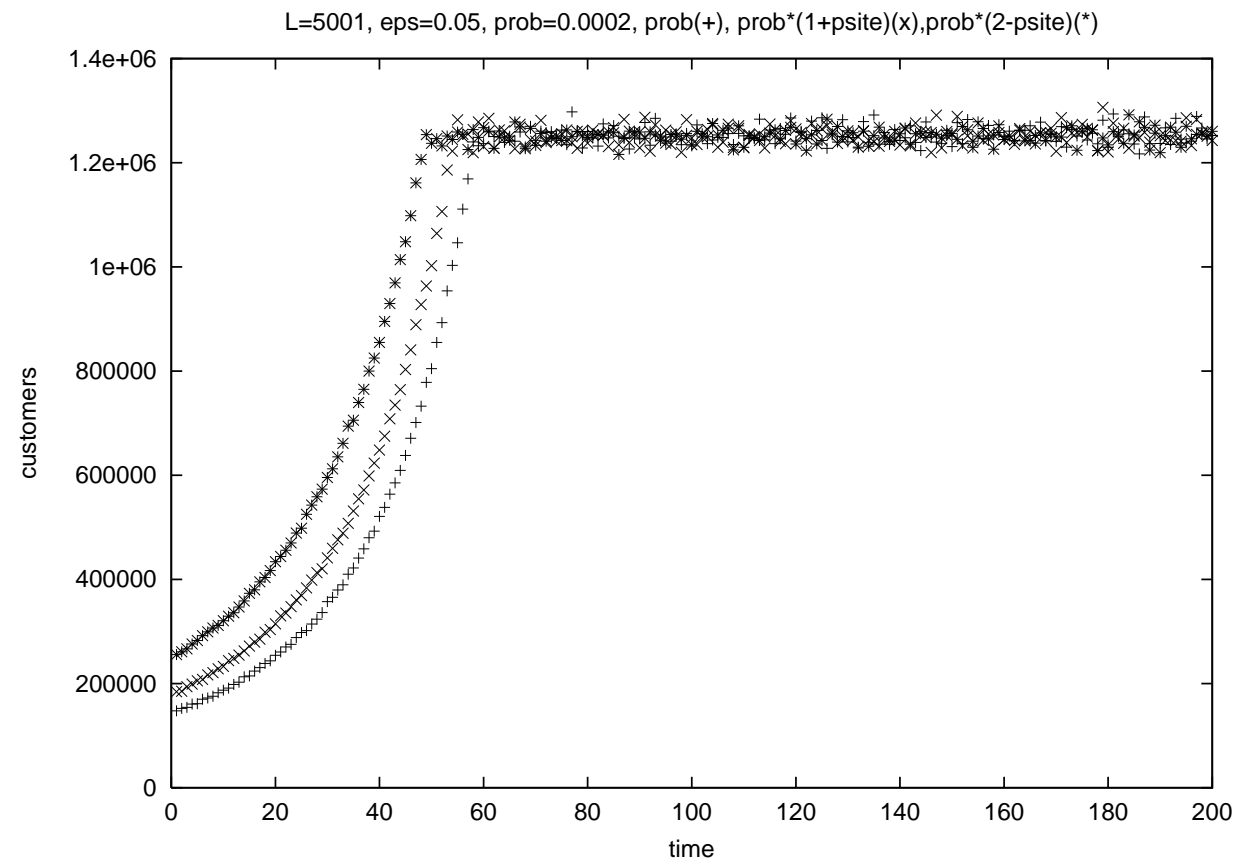

Figure 4: Comparison of homogeneous influence $h$ of advertising $(+$; as in Figs. 1 and 2) with an individual advertising success $\left(1+p_{i}\right) h(\mathrm{x})$ and $\left(2-p_{i}\right) h$ $(*)$, averaged over 20 lattices $5001 \times 5001$ at $h=0.0002, \epsilon=0.05$.

\section{References}

[1] S. Solomon and G. Weisbuch, adap-org 9909001

[2] S. Solomon, G. Weisbuch, L. de Arcangelis, N. Jan and D. Stauffer. Physica A 277, 239 (2000)

[3] C. Moukarzel, private communication, 2000, and remarks at the 5th Conference on Social Simulation, Polands, Sept. 2001.

[4] Z.F. Huang, Eur.Phys.J. B 16, 379 (2000); E. Ahmed and H.A. Abdusalam, Eur.Phys.J. B 16, 569 (2000); G. Weisbuch and D. Stauffer, Physica A 287, 563 (2000); A. KarGupta and D. Stauffer, Int.J.Mod.Phys. C 11, 695 (2000); G. Weisbuch and S. Solomon, Int.J.Mod.Phys. C 11, 1263 (2000); J. Goldenberg, B. Libai, S. Solomon, N. Jan, and D. Stauffer, Physica A 284, 335 (2000) 
[5] Z.F. Huang, Int.J.Mod.Phys. C 11, 287 (2000)

[6] A. de Vany and D. Wall, Economic J. 106, 1493 (1996). 